\title{
Utilización de colgajo radial para cobertura de exposición protésica en craneoplastias
}

\author{
Use of radial flap to cover prosthetic exposure in cranioplasty
}

\author{
Autora: Daniela Belén Rocca'. Coautores: Marcelo Mackfarlane², Guillermo Artero², \\ Mónica Martínez ${ }^{1}$, Joaquín Pefaure ${ }^{2}$
}

\section{RESUMEN}

Introducción. La craneoplastia es un procedimiento necesario para cubrir defectos craneales luego de resección ósea por distintas etiologías, tales como hemorragia intracraneal, traumatismos craneoencefálicos, tumores o infecciones. Una de las complicaciones frecuentes es la exposición de placas de craneoplastia por dehiscencia de herida cutánea. Estas son complicaciones frecuentes y frustrantes para el paciente y el cirujano plástico. La transferencia de tejidos a distancia brinda una solución para estos pacientes. El colgajo radial antebraquial reúne las condiciones necesarias para la cobertura.

Material y métodos. Se realiza un estudio retrospectivo con un total de 14 pacientes en el Hospital Santojanni en el período comprendido entre enero de 2018 y marzo de 2020. Todos presentaron exposición de la placa de craneoplastia. Se analizó el área de defecto, siendo el área promedio a cubrir de $5,07 \mathrm{~cm} 2$ (1,5-12,8 cm2). Se realiza cobertura con colgajo radial antebraquial. Se utilizan vasos faciales homolaterales (57\%) como primera elección; vasos faciales contralaterales, por radioterapia (29\%) y en ellos se utilizó bypass en un tiempo con interposición venosa en tres casos y arterial en el restante; vasos temporales superficiales (14\%).

Resultados. Se logró cobertura completa en todos los pacientes. La vitalidad de los colgajos fue del $100 \%$. Seguimiento promedio de 12 meses (4-23 meses). Un paciente presentó seroma en la zona dadora. No se presentaron nuevas exposiciones ni dehiscencias.

Conclusiones. La transferencia con tejido a distancia permite una eficaz cobertura de material expuesto. El colgajo antebraquial proporciona tejido blando confiable, delgado y bien vascularizado, que se puede utilizar para sellar la duramadre, eliminar el espacio muerto, cubrir el defecto expuesto y también posee un pedículo largo que permite anastomosis a distancia en casos de defectos tratados con radioterapia.

Palabras claves: complicaciones de craneoplastias, reconstruccion de cuero cabelludo, colgajo radial.

\begin{abstract}
Background. Cranioplasty is a procedure that provides coverage for cranial defects after bone resection because of different etiologies such as intracranial hemorrhage, trauma, tumor or infection. One of the most important postoperative complications is the exposure of the plate, that may happen after a skin wound dehiscence. These are challenging situations for the plastic surgeon. Free tissue transfer provides a solution for these patients. The forearm radial flap provides all the conditions to solve these problem

Methods. A retrospective study was performed with fourteen patients at the Santojanni Hospital between January 2018 and March 2020. All of them presented exposure of the cranioplasty plate. The defect area was analyzed. The average area to be covered was $5.07 \mathrm{~cm} 2(1.5 \mathrm{~cm} 2-12.8 \mathrm{~cm} 2)$. A radial forearm free flap was performed for all patients. Homolateral facial vessels (57\%) were used as the first choice; the contralateral facial vessels were used in case of previous radiation therapy (29\%) and in these cases a bypass was used in one case with venous interposition in three cases and arterial in the rest; superficial temporal vessels (14\%).

Results. Flap vitality was $100 \%$. Average follow-up of 12 months ( $23 \mathrm{~m}-4 \mathrm{~m})$. One patient presented seroma in the donor area. No new exposures or dehiscences were presented.

Conclusions. Free tissue transfer provides an effective coverage to exposed material. The forearm flap provides reliable, thin, well-vascularized soft tissue that can be used to seal the dura, remove dead space, cover the exposed defect, not only but also it provides a long pedicle that allows distant anastomosis in cases of radiation therapy.
\end{abstract}

Keywords: cranioplasty complications, scalp reconstruction, radial flap.

\section{INTRODUCCIÓN}

Los defectos craneales complejos suelen ser resultado de una amplia variedad de etiologías como, por ejemplo, trauma, tumores, hemorragia intracraneal de diversas causas, malformaciones congénitas e intervenciones neuroquirúrgicas varias ${ }^{1}$.

Cuando no se puede reconstruir la arquitectura original del cráneo, estos defectos requieren de una craneoplas-

1. Residente Cirugía Plástica y Reconstructiva, Hospital Donación F. Santojanni.

2. Cirujano Plástico, Servicio de Cirugía Plástica, División de Cirugía, Hospital Donación F. Santojanni.

$\square$ Correspondencia: revista@sacper.org.ar

Los autores no declaran conflictos de intereses

Recibido: 09/05/2020 / Aceptado: 12/05/202 tia. Esta cirugía se encarga de reestablecer la cobertura con objetivo de proteger el contenido intracraneal, evitando complicaciones secundarias como la atrofia cerebral y consecuentes convulsiones, como también devolverle la estética del contorno craneofacial al paciente y proveerle de soporte a los tejidos blandos craneofaciales ${ }^{2,3}$.

Diversos materiales son los que se utilizan en las craneoplastias incluidos hueso autólogo, mallas de titanio y polimetilmetacrilato (PMMA). El hueso autólogo es comúnmente utilizado en niños para prevenir anomalías relacionadas con el crecimiento; se conoce de este material una resistencia superior a la infección y posterior extrusión, pero por otro lado es más propenso a sufrir resorción ósea y también morbilidades en la zona dadora $^{4,5}$. Las mallas de titanio son biocompatibles y radiolúcidas. PMMA es un material radiolúcido, liviano, maleable y el más utilizado por muchos autores. Estos dos últimos materiales protésicos son los más empleados a la hora de realizar craneoplastias ${ }^{3}$. 
TABLA1. CASUISTICA.

\begin{tabular}{|c|c|c|c|c|c|c|}
\hline $\mathrm{N}^{\circ}$ & Edad/Sexo & $\begin{array}{l}\text { Colgajo local } \\
\text { fallido }\end{array}$ & $\begin{array}{l}\text { Material del } \\
\text { implante }\end{array}$ & Área expuesta & $\begin{array}{l}\text { Tiempo desde la coloca- } \\
\text { ción hasta su exposición }\end{array}$ & $\begin{array}{c}\text { Defecto de co- } \\
\text { bertura } \mathrm{cm}^{2}\end{array}$ \\
\hline 1 & $78 / \mathrm{H}$ & & Titanio & Temporoparietal, d & $3 \mathrm{~m} 23 \mathrm{~d}$ & $1,4 \times 2,2$ \\
\hline 2 & $48 / \mathrm{M}$ & & Titanio & Temporoparietal, d & $6 \mathrm{~m}$ & $2,4 \times 3$ \\
\hline 3 & $28 / \mathrm{H}$ & & Titanio & Parietal, I & $2 \mathrm{~m} 14 \mathrm{~d}$ & $1,5 \times 1,5$ \\
\hline 4 & $56 / \mathrm{H}$ & sí & Titanio & Temporoparietal, I & $3 \mathrm{~m}$ & $4 \times 3,2$ \\
\hline 5 & $67 / \mathrm{H}$ & & PMMA & Temporoparietal I & $3 \mathrm{~m}$ & $2,5 \times 2$ \\
\hline 6 & $53 / F$ & & Malla de titanio & Frontal & $4 \mathrm{~m}$ & $3 \times 4$ \\
\hline 7 & $37 / \mathrm{H}$ & & Titanio & Temporoparietal, D & $5 \mathrm{~m}$ & $2,6 \times 3,5$ \\
\hline 8 & $55 / M$ & & Titanio & Frontal & $7 \mathrm{~m}$ & $3,5 \times 3,2$ \\
\hline 9 & $30 / \mathrm{H}$ & sí & Titanio & Temporoparietal, D & $2 m$ & $232 \times 3,2$ \\
\hline 10 & $19 / M$ & & PMMA & Temporoparietal, I & $3 \mathrm{~m}$ & $2,3 \times 3$ \\
\hline 11 & $34 / \mathrm{H}$ & & PMMA & Temporoparietal, D & $4 \mathrm{~m}$ & $1,5 \times 2$ \\
\hline 12 & $39 / \mathrm{H}$ & & Titanio & Frontal & $1 \mathrm{~m} 29 \mathrm{~d}$ & $1,5 \times 1$ \\
\hline 13 & $18 / \mathrm{H}$ & & Titanio & Parietal, I & $4 \mathrm{~m}$ & $2,4 \times 1$ \\
\hline \multirow[t]{2}{*}{14} & $49 / M$ & & Malla de titanio & Temporoparietal, D & $3 m$ & $1,2 \times 2,5$ \\
\hline & 40,07 & & & & & 5,97 \\
\hline
\end{tabular}

La incidencia de complicaciones de las craneoplastias varía según los autores entre un $12 \%$ y hasta un $50 \%$,79. Estas están fuertemente asociadas a la persistencia de patología residual, a la cantidad de procedimientos quirúrgicos a los cuales se somete el paciente previo a la realización de la craneoplastia y, por último, al material de reconstrucción. El hueso autólogo presenta menor incidencia de complicaciones, pero esta es mayor a nivel del sitio dador, y tienen que ser consideradas?. Dentro de las complicaciones posoperatorias tempranas se presentan dehiscencia de herida, hematoma, infección y necrosis de los colgajos cutáneos. Dentro de las complicaciones frecuentes tardías, exposición de placa, deformidades, resorción del material protésico. La complicación de exposición de placa presenta una incidencia del $11 \%{ }^{9}$. Por lo general, se da a nivel de la cicatriz, en donde la herida presenta mayor tensión. Esta complicación se atribuye entre otros factores de riesgo, además de los nombrados anteriormente, a la viabilidad de los colgajos cutáneos a la hora del cierre. Otro de los factores de riesgo es el material protésico. Este genera una presión a nivel de los tejidos blandos que, en ocasiones, termina en úlcera y exposición. Hay diferentes formas de abordar estos pacientes, que se concluyen en extracción de la placa y cobertura, o no extraerla y realizar una cobertura con tejido apropiado, bien vascularizado y con una buena calidad de tejidos blandos ${ }^{3,7,9}$. En este trabajo nos proponemos un abordaje del paciente conservador, ya que la extracción de la placa presenta implicancias psicológicas, económicas y estéticas de interés en el paciente. Por esta razón proponemos cobertura con colgajo radial libre. Nuestro objetivo es demostrar que, mediante la transferencia libre de tejido, podemos tratar este tipo de pacientes ${ }^{21,22}$.

\section{MATERIAL Y MÉTODOS}

Este es un estudio retrospectivo, realizado en el Hospital Donación Francisco Santojanni en el período enero de 2018 a marzo de 2020 . Se analizaron en total 14 (catorce) pacientes de los que 10 (diez) eran hom- bres y 4 (cuatro), mujeres, con un promedio de edad de 40 años (el menor de 18 años y el mayor de 56). Todos presentaron exposición de la craneoplastia. Dos de ellos acuden derivados de otra institución en donde se les realizó una primera cobertura fallida con colgajo regional (Tabla 1). Se detalla en esa tabla el material de craneoplastia así como el área de exposición. Se evalúa el tamaño del defecto de cobertura mediante medición con centímetro del área expuesta: promedio de $5,97 \mathrm{~cm}^{2}$ (la mayor de $12,8 \mathrm{~cm}^{2}$ [paciente 4] y la menor de 1,5 [paciente 12]). Se propone para estos pacientes cobertura con colgajo libre antebraquial. Se realiza a todos los pacientes como método diagnóstico test de Allen y eco-Doppler de vasos.

Se debe comenzar realizando una historia clínica detallada del paciente, haciendo hincapié en historia de cirugías previas, motivo de la colocación del material protésico, tiempo de exposición de la misma e interrogar sobre mano hábil. También se deben tener en cuenta factores de riesgo como diabetes, obesidad, hipertensión, edad. Luego se debe realizar un examen físico minucioso evaluando la existencia de incisiones o cicatrices previas a nivel craneofacial, la presencia de tatuajes y cicatrices en la zona antebraquial bilateral, la presencia de parálisis facial, la palpación del pulso a nivel de los vasos receptores temporales superficiales, faciales homolaterales y faciales contralaterales. Otra de las variables a medir es el área del defecto de cobertura, teniendo en cuenta también el área de tejido comprometido en la zona afectada que se encuentra desvitalizado y/o con fibrosis, que será resecado durante la cirugía. Para ello utilizamos una cinta métrica y calculamos el área multiplicando la base del defecto por la altura. Se prosigue realizando el test de Allen. Este se realiza para asegurarse de que la vascularización de la mano puede mantenerse indemne una vez seccionada la arteria radial. Se utiliza para poner en evidencia la existencia de variaciones anatómicas que puedan llegar a comprometer la vasculartura de la mano. Se comienza realizando una palpación de la arteria radial y de la arteria cubital a nivel de la muñeca. Se le pide al paciente que 


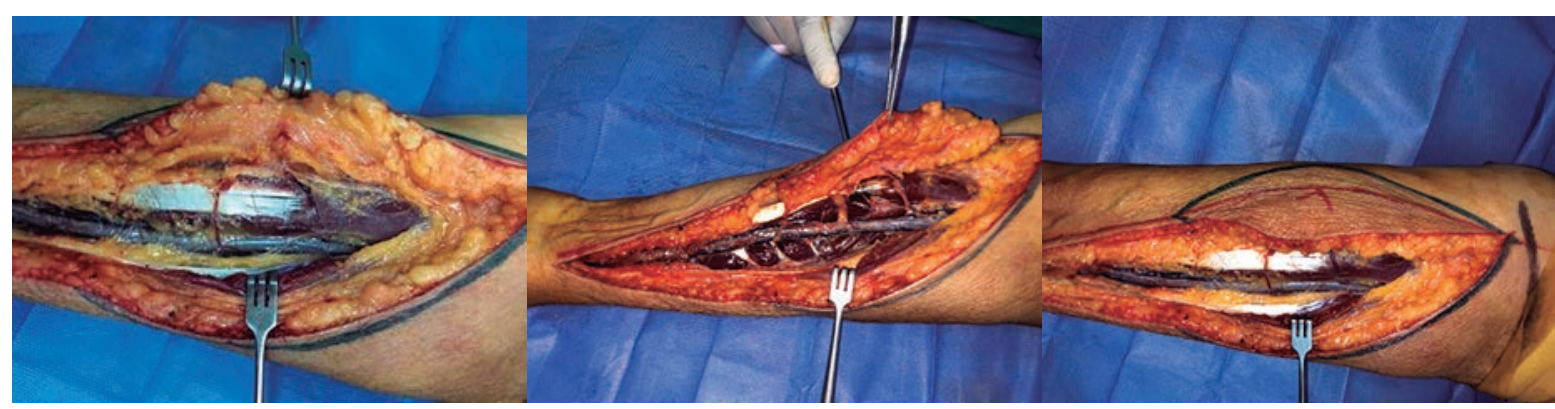

Figura 1.

comprima la mano, el cirujano luego comprime ambas arterias con igual presión. Una vez que esto sucede, se le pide al paciente que extienda los dedos, sin hacer una hiperextensión. Después estas maniobras, aún con la compresión de ambas arterias la mano debería tornar de color blanquecino. Una vez que esto sucede, se mantiene presionada la arteria radial y se suelta la cubital. Inmediatamente la mano debería recuperar su vascularización y tornarse de coloración rosada ${ }^{13}$. El paso siguiente es realizar un eco-Doppler de vasos temporales para evaluar el flujo.

\section{TÉCNICA QUIRÚRGICA (Figura 1)}

Se comienza realizando la marcación del colgajo radial del antebrazo. Se selecciona el antebrazo no dominante, con test de Allen normal. Se marca el trayecto de la arteria radial trazando una línea que comienza $1 \mathrm{~cm}$ distal al centro de la fosa antecubital hasta el tubérculo del escafoides. Esto, además de marcar el curso de la arteria radial marca la ubicación del tabique intermuscular lateral, que la incorpora junto con las venas comitantes. La arteria es fácilmente palpable en el tercio distal del antebrazo. Luego se procede a marcar la perforante, previamente seleccionada con Doppler. Por lo general diseñamos los colgajos a nivel del antebrazo distal, donde la piel es mas fina y se encuentra más cantidad de perforantes. Luego se toman las medidas del defecto a cubrir. Esto se utiliza como referencia para marcación y tallado del colgajo. Una vez realizado esto se coloca al paciente en decúbito dorsal, con extensión del brazo no hábil a $90^{\circ}$ en una mesa de mano, y el brazo totalmente supinado. Se realiza luego un torniquete con una venda smarch de $2 \mathrm{~m} \times 10 \mathrm{~cm}$ en la raíz del brazo, justo por debajo de la axila, con la compresiónn necesaria. Esto permite una disección prolija, con mejor visualización por ausencia de sangrado.

Se trabaja con 2 equipos quirúrgicos para optimizar las tiempos de cirugía. Un equipo se coloca a la altura de la cabeza del paciente para realizar la disección de los vasos receptores. El segundo equipo quirúrgico realiza la disección del colgajo radial.

Para la disección del colgajo radial, el cirujano se coloca en posición sentado del lado cubital del paciente y comienza realizando la incisión también del lado cubital. Esta incisión se realiza hasta la grasa subcutánea y se identifican las venas subcutáneas proximales y los nervios sensitivos superficiales a preservar. Comenzamos la elevación de este colgajo suprafascial preservando la fascia. Esto ayuda a proteger los músculos y tendones flexores, y trata de disminuir la morbilidad de la zona dadora disecando radialmente sobre el palmar largo. El objetivo es ubicar a la perforante. Una vez que esta se ubica, se profundiza el plano de disección a través de la fascia, de modo que un manguito de fascia que rodea el pedículo vascular se mantiene intacto. Se procede a seguir hasta la perforante hasta la arteria radial, la cual se ubica a nivel proximal por debajo del músculo braquirradial y a nivel distal entre este y el palmar largo. Se continúa realizando una ligadura de las ramas de la arteria radial que son numerosas con el uso de ligaclip 100 y microclip. Una vez que se obtiene el colgajo con su perforante y el pediculo de longitud necesaria, se procede a realizar la ligadura con ligaclip de la arteria radial y de las venas comitantes.

Es necesario hacer algunas aclaraciones. La disección suprafascial no produce un colgajo significativamente más delgado. Está dirigido principalmente a mejorar el defecto del sitio donante.

Es importante identificar la rama cutánea del nervio radial y preservarla intacta para evitar la pérdida posterior de la sensibilidad o los neuromas dolorosos.

El otro equipo quirúrgico ya tiene preparado los vasos receptores para recibir el colgajo.

El abordaje quirúrgico del cuello depende de si se realizó o no una disección del cuello. Si es así, el acceso es simple. Si no es así, y si se está abordando el cuello únicamente para el acceso a los vasos, se prefiere una incisión transversal a lo largo de uno de los pliegues naturales de la piel. Si los vasos temporales están indemnes, se realiza una incisión tipo lifting preauricular para ubicarlos. La disección ocasionalmente debe extenderse proximalmente al despegue de la rama profunda, para obtener el mayor calibre; sin embargo, si el calibre de los vasos más distalmente es adecuado, se puede realizar una anastomosis directa.

Como ventaja, la arteria temporal superficial tiene facilidad de acceso. Una incisión preauricular simple proporciona un fácil acceso tanto a la arteria como a la vena. La proximidad de estos vasos al cuero cabelludo y a la cara media los hace ideales para revascularizar los colgajos; sin embargo, los vasos son bastante susceptibles al espasmo y la vena tiende a ser de paredes del- 


\section{VASOS RECEPTORES}

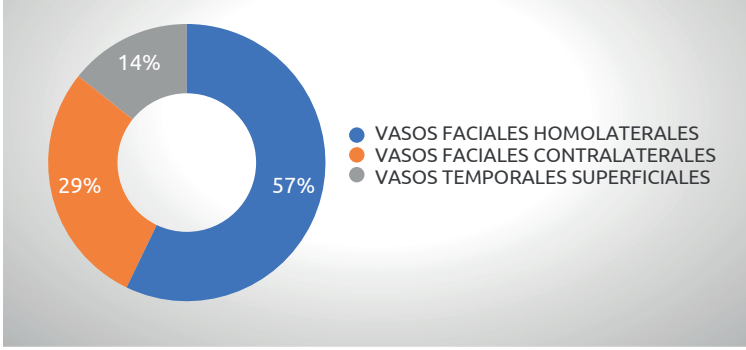

Figura 2.

gadas y friables. En términos de calibre, con frecuencia es necesario diseccionar los vasos de regreso a la región pretragal donde se desprende la rama profunda de la arteria temporal superficial. Proximal a esta rama, el calibre de la arteria y la vena es superior.

De lo contrario, se busca en cuello la arteria facial, la cual al ser rama directa de la arteria carótida externa presenta buen diámetro, alto flujo y presión de perfusión aceptable. Es de gran ayuda la palpación en el borde inferior de la mandíbula. Se realiza una corta incisión transversal en el cuello, debido a que este vaso está ubicado debajo del borde de la mandíbula; durante el acceso, debe diseccionarse bien hacia su origen y, a veces, debe bajarse por debajo del vientre posterior del músculo digástrico. Debe tenerse sumo cuidado y ubicar la rama marginal mandibular del nervio facial para no lesionarlo. La vena facial anterior está ubicada anterior a la arteria facial y, aunque la arteria es algo tortuosa, la vena es relativamente recta, tiene buen calibre y es muy útil como receptor para una transferencia de tejido libre. En algunas ocasiones la vena facial anterior se divide en pacientes que han tenido una disección del cuello y, por lo tanto, no está disponible. En caso de tener que cubrir un defecto más alejado al de la zona expuesta utilizamos injerto de vena safena, por no tener un calibre demasiado grande y limitar su capacidad de dilatación.

Se realiza una incisión longintudinal en cara interna de pierna, se disecan los planos y una vez encontrada la vena se extrae el largo necesario a utilizar. Luego, realizamos lavado con solución fisiológica por el lumen. Una vez preparado lo utilizamos en sentido inverso para la revascularización. La sutura se realiza en forma término-lateral para disminuir el riesgo de trombosis. Este procedimiento puede extender la duración de la operación, pero simplifica considerablemente la realización de las suturas vasculares.

Una vez que tenemos el colgajo y los vasos receptores listos, utilizamos heparina $3000 \mathrm{U}$ endovenosa y esperamos 3 minutos para ligar la arteria radial y trasladar el colgajo a la zona receptora. Esto mantiene una presión de perfusión, reduce la viscosidad y aumenta el diámetro del vaso del pedículo. Pasado este tiempo se realiza ligadura distal y se secciona la arteria radial dejando un clamp proximal.
Bajo visión microscópica 16X se procede a la anastomosis de los pedículos. Los vasos son irrigados con suero heparinizado. Esto no aumenta el riesgo de hematoma ni hemorragia posoperatoria. Preferentemente, se realiza anastomosis término-lateral, para dar mejor llegada de flujo a los vasos, o término-terminal. Se colocan clamps en el pedículo receptor y donante. En primera instancia se realiza la sutura de la vena con hilo nylon 8.0 mediante puntos simples. En segundo lugar se realiza la anastomosis arterial. Una vez realizada la anastomosis, se comprueba la vitalidad del colgajo y se realiza el modelado del colgajo acorde al defecto a cubrir. Para cerrar, se realiza una sutura de puntos separados con nylon 3.0. Se ofrece un drenaje al lecho.

Prácticamente, toda la piel de la zona dadora del antebrazo desde el codo hasta la muñeca se puede tomar como un colgajo radial del antebrazo, pero esto interrumpiría severamente el drenaje linfático de la mano. En la práctica, una tira de piel de al menos 3 $\mathrm{cm}$ de ancho que recubre el compartimento extensor posterior del antebrazo y el borde subcutáneo cubital debe mantenerse intacto. Esta es también el área que tiene la vascularización más pobre en función de la arteria radial y las venas de drenaje. Para cubrir el área dadora, utilizamos un injerto de piel parcial que tomamos de la piel del muslo anterior ipsilateral. Realizamos la curación con un apósito de Brown, el cual revisamos a los 5 días. Se realiza un control posoperatorio evaluando la vitalidad del colgajo mediante el relleno capilar, prestando atención a la eventual presencia de hemantomas ${ }^{10,14}$.

Como vasos receptores se utilizaron los vasos faciales homolaterales de forma electiva en 8 pacientes $(57 \%$ de los casos); vasos temporales superficiales en 2 pacientes ( $14 \%$ de los casos); vasos faciales contralaterales cruzados, por radioterapia, en 4 pacientes (29\% de los casos). En estos fue necesaria la utilización de bypass, realizado en un tiempo quirúrgico, con interposición venosa en tres casos y arterial en el restante ${ }^{30}$ (Figura 2).

\section{RESULTADOS}

El tiempo quirúrgico promedio fue de 3 horas $40 \mathrm{mi}$ nutos. El tiempo de estadía hospitalaria promedio fue de 5 días. La vitalidad de los colgajos fue del 100\%. Se realizó una revisión de colgajo en el posoperatorio inmediato por hematoma, el cual se resolvió sin ninguna complicación. En el resto de los pacientes no se registraron complicaciones. El seguimiento promedio fue de 12 meses, para un máximo de 23 meses y un mínimo de 4 meses. El cierre del área dadora fue con injerto de piel parcial tomado de la piel del muslo anterior de la pierna homolateral al colgajo radial en ocho de los casos. En seis casos se pudo realizar el cierre directo. 

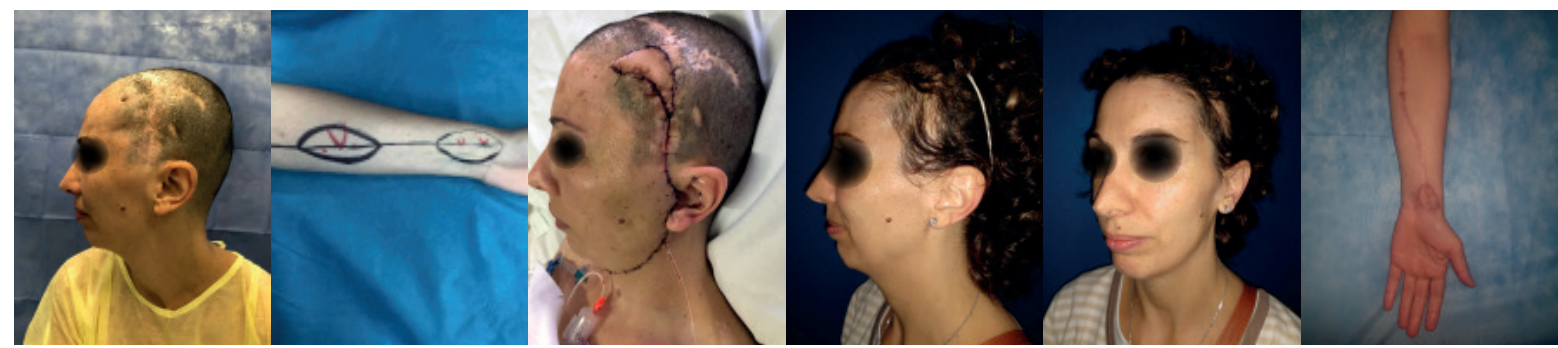

Figura 3. Caso 1.

\section{DISCUSIÓN}

La exposición de las placas de craneoplastia es un verdadero desafío reconstructivo para el cirujano plástico. Cuando se expone una placa en primera instancia, muchos autores recomiendan la cobertura con colgajos locales o regionales, y se han descripto resultados satisfactorios con dichos procedimientos ${ }^{15,16}$. Sin embargo, cuando un colgajo local o regional, en el afán de salvar una placa de craneoplastia, falla, por lo general da como resultado un defecto de cobertura mucho mayor que el que se tenía en un principio. Por lo tanto, genera un terreno más complejo a tratar ${ }^{17}$. En dos de los pacientes de nuestro estudio se realizó el colgajo radial en segunda instancia, luego de un primer intento de cobertura con colgajos locales en otra institución. En ambos casos se trató de colgajos de avance que terminaron complicándose con dehiscencia y exposición de placa. En el caso de nuestro paciente número 12, con un área de defecto de $1,5 \mathrm{~cm}^{2}$, un cirujano plástico puede verse tentando en realizar un colgajo local para cobertura. Sin embargo, existen ciertos factores que podrían explicar este fenómeno, y el motivo por el cual elegimos realizar un colgajo libre en lugar de un colgajo local a pesar del tamaño pequeño del defecto a cubrir. En primer lugar, la circulación en los tejidos blandos periplaca por lo general se encuentra afectada. Es importante resaltar que las incisiones que se realizan en la región temporoparietal deben respetar la vascularización del cuero cabelludo. En ciertos casos, si no se respe$\tan$ los vasos temporoparietales, los colgajos suelen tener sufrimiento y dehiscencia posterior ${ }^{18}$. Esta circulación se encuentra afectada ya sea por la existencia de tejido cicatrizal debido a cirugías previas, que a veces suelen ser múltiples; radioterapia, cuando se trata de causas oncológicas; la historia de traumatismo de cráneo, que en nuestro caso es la etiología de mayor incidencia de craneoplastias; o el cierre, que muchas veces se produce con tensión, aumentando la presión que genera la placa sobre los tejidos blandos, cuyo resultado es atrofia de los mismos, ulceración y extrusión de la pla$\mathrm{ca}^{19}$. Por ello, a pesar de que consideramos la escalera reconstructiva, una estructura importante a la hora de la toma de decisiones, en estos casos preferimos directamente realizar la cobertura con una transferencia libre de tejido. Con la utilización de colgajos libres se logra aumentar la irrigación del área gracias a su condi- ción de ser ampliamente vascularizado. Este aumento de vascularización en la zona genera un efecto de "antiinfección" adicional; la infección muchas veces es la causa de la dehiscencia o extrusión de la placa ${ }^{27}$. Sin embargo, este último concepto está ampliamente discutido y poco demostrado en trabajos científicos.

Otro de los puntos de discusión es la elección adecuada del colgajo libre. Ciertos autores nombran al colgajo anterolateral del muslo y al colgajo dorsal ancho como workhorse flap en el tratamiento de grandes defectos de cuero cabelludo ${ }^{20}$. La mayoria de series y reportes de casos publicados utilizan el colgajo anterolateral del muslo para los defectos complejos; sin embargo, no hay publicaciones de estudios de investigación randomizados ${ }^{39,40}$. El colgajo antebraquial, publicado en el año 1981 por los doctores Yang Guofan y Gao Yuzhi, está basado en perforantes de la arteria radial. Desde esa época ya se hablaba de su utilidad para reconstrucción de quemaduras de cabeza y cuello ${ }^{14}$. Es un colgajo que reúne caracteristicas fundamentales para reconstruir este tipo de defectos. Por un lado ofrece una cobertura con tejido blando fino, flexible y con gran variabilidad en tamaño, suficiente para los defectos de nuestros pacientes en cuestión. En cuanto al drenaje venoso, nos permite utilizar venas comitantes profundas y superficiales, brindando de este modo la posibilidad de aumentar el flujo de drenaje, dada las condiciones de alto flujo arterial que posee el mismo. Por último, y no menos importante, presenta un pedículo vascular largo, lo que nos permite usar vasos receptores a distancia de la zona cruenta. El tema de los vasos receptores es otra cuestión a discutir. Los vasos temporales superficiales son generalmente los más cercanos al áera en cuestión. Muy frecuentemente se encuentran afectados por la historia de traumatismo y cirugías previas que presentan nuestros pacientes. Es de suma importancia tener este concepto presente desde el momento de la primera cirugía a la cual se somete el paciente, ya que preservar los vasos temporales superficiales es de suma importancia ${ }^{19}$. Esta frecuente afección genera un desafío a la hora de decidir cuáles son los vasos más aptos para brindar vascularización adecuada al colgajo. Para identificar si existe un correcto flujo de los vasos temporales, es conveniente realizar un Doppler color con medición del diámetro y velocidad del flujo ${ }^{30,32-34}$. De no ser viable la velocidad de flujo, 


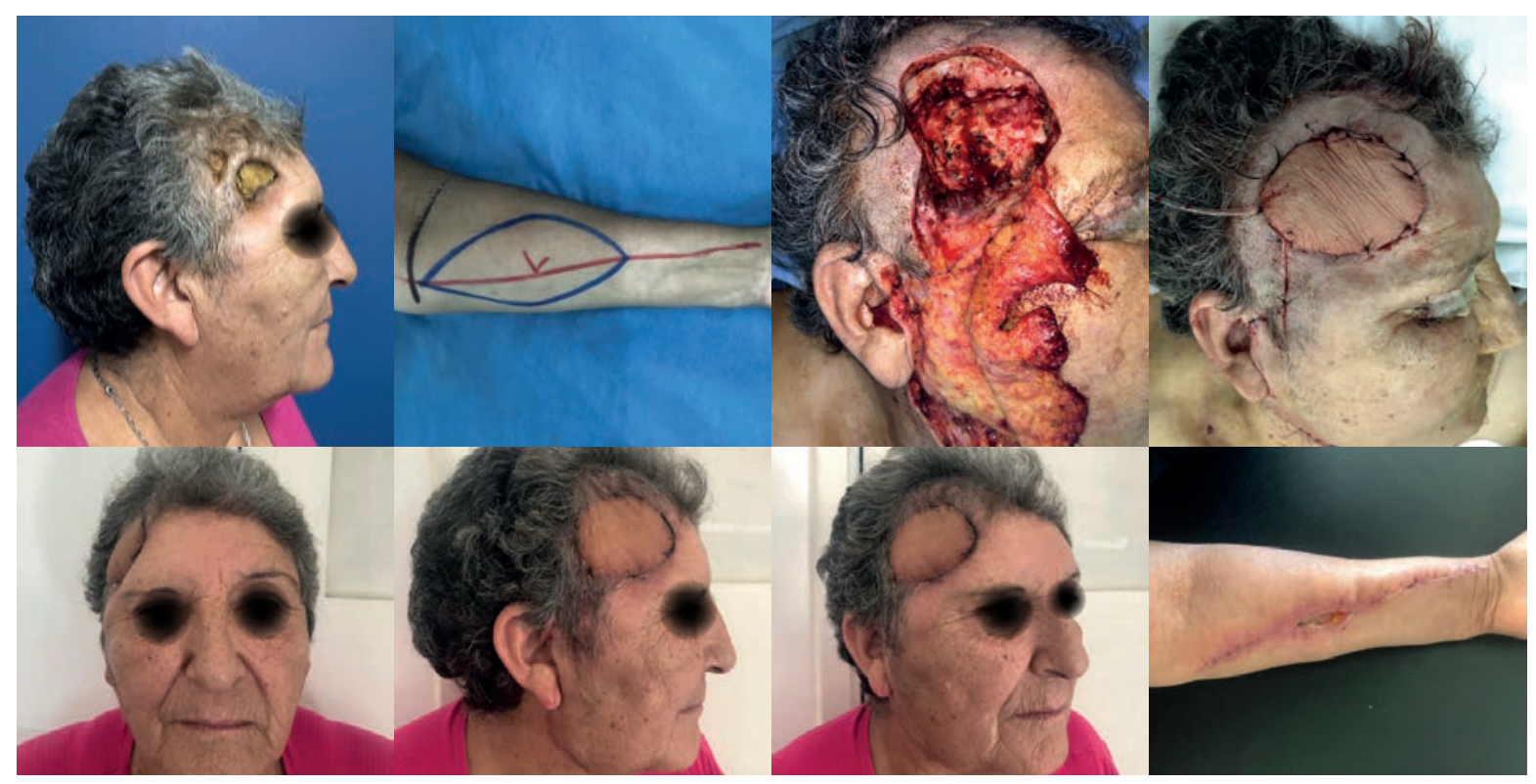

Figura 4. Caso 2.
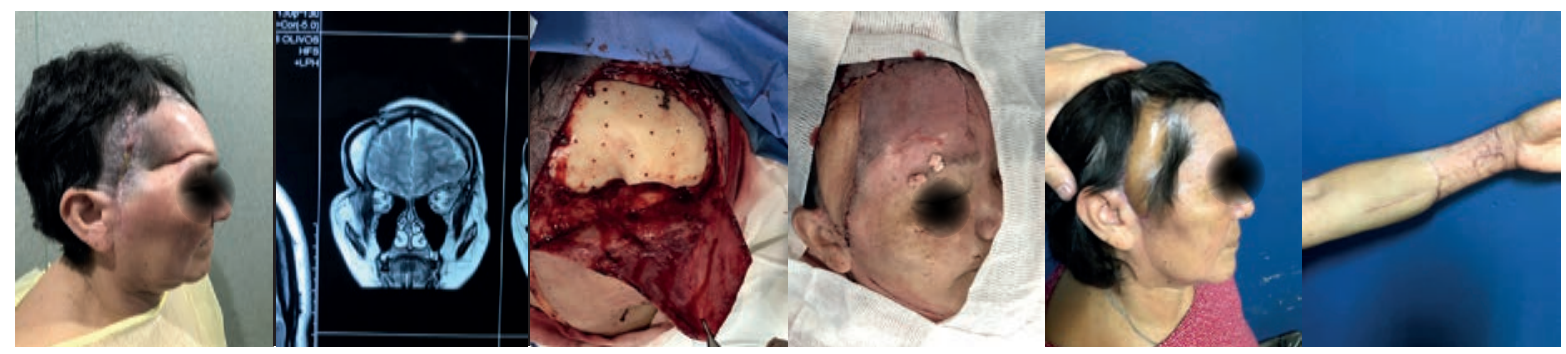

Figura 5. Caso 3.

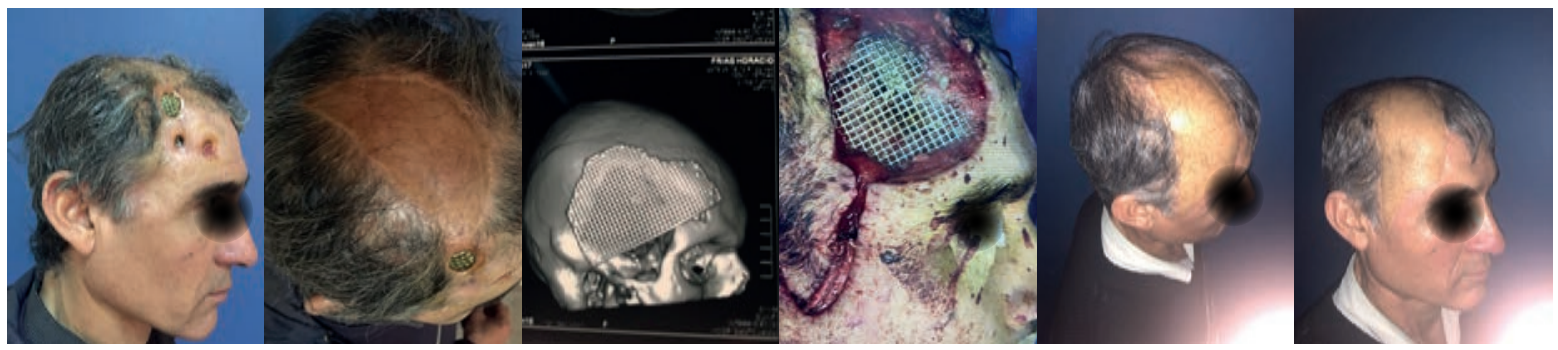

Figura 6. Caso 4

la segunda opción es utilizar los vasos faciales homolaterales al origen de la artreria facial. Como tercera opción, en caso de ser irradiada la zona facial homolateral, se debe tomar la decisión de ir a los vasos faciales contralaterales o temporales contralaterales. Esto se logra mediante la interposición venosa o arterial. Estos son los tres conceptos tenidos en cuenta por el servicio a la hora de elegir al colgajo radial para la cobertura de la zona cruenta.

\section{CASO 1}

Paciente femenino con exposición de placa en región temporal. Se realiza colgajo antebraquial con anastomosis a nivel de vasos faciales, zona dadora con secuela mínima (Figura 3).

\section{CASO 2}

Paciente femenino con exposición de placa en región temporal. Se realiza colgajo antebraquial con pastilla de piel próxima con perforarantes proximales, zona dadora con cierre en primera instancia (Figura 4).

\section{CASO 3}

Paciente femenino con exposición protésica temporal, colgajo distal, con injerto de piel en zona dadora (Figura 5).

\section{CASO 4}

Paciente masculino tratado con injerto de piel. Se realiza colgajo antebraquial con bypass arteriovenoso, con vena safena para cobertura de gran defecto (Figura 6). 


\section{CONCLUSIÓN}

En casos de defectos de cobertura cutáneos secundarios a exposición de material protésico por cranioplastia, es necesaria la cobertura con un tejido bien vascularizado, que generalmente corresponde a la transferencia libre. Es necesario identificar el pedículo vascular receptor previo a la toma de la decisión de la transferencia. Los vasos faciales homolaterales son la prioridad para dicha transferencia. El colgajo antebraquial a flujo directo o reverso cumple las necesidades para dicha cobertura. Es prioritaria la cobertura de toda la unidad estética para obtener mejores resultados estéticos. No está claro si debe resecarse o no el material protésico en el primer tiempo ya que es posible que la transferencia de tejido libre vascularizado actúe sellando el espacio entre el material protésico y tejidos blandos.

\section{BIBLIOGRAFÍA}

1. Stephens FL, Mossop CM, Bell RS, et al. Cranioplasty complications following wartime decompressive craniectomy. Neurosurg Focus. 2010;28(5):E3.

2. Dujovny M, Aviles A, Agner C, Fernandez P, Charbel FT. Cranioplasty: cosmetic or therapeutic? Surg Neurol 1997:47(3):238-41.

3. Reddy $S$, Khalifian S, Flores JM, et al. Clinical outcomes in cranioplasty: risk factors and choice of reconstructive material. Plast Reconstr Surg 2018

4. Manson PN, Crawley WA, Hoopes JE. Frontal cranioplasty: Risk factors and choice of cranial vault reconstructive material. Plast Reconstr Surg 1986;77:888-904.

5. Yadla S, Campbell PG, Chitale R, et al. Effect of early surgery, material, and method of flap preservation on cranioplasty infections: $A$ systematic review. Neurosurgery 2011;68:1124-9; discussion 1130.

6. Sahoo NK, Tomar K, Thakral A, Rangan NM. Complications of Cranioplasty. J Craniofac Surg 2018:29(5):1344-8

7. Grant FC, Norcross NC. Repair of cranial defects by cranioplasty. Ann Surg 1939;110:488-512.

8. Chang V, Hartzfeld P, Langlois M, Mahmood A, Seyfried D. Outcomes of cranial repair after craniectomy. J Neurosurg 2010;112:1120-4.

9. De Bonis P, Frassanito P, Mangiola $A$, et al. Cranial repair: how complicated is filling a "hole"? J Neurotrauma 2012;29:1071-6.

10. Craniofacial, Head and Neck Surgery and Pediatric Plastic Surgery. Vol 3. In: Rodriguez E, Losee J, Neligan P. Plastic Surgery 4th Edition. 2017.

11. Chang D. Microvascular Reconstruction of the Skull Base. Semin Surg Oncol 2000:19:211-7.

12. Evans GR, Schusterman MA, Kroll SS, et al. The radial forearm free flap for head and neck reconstruction: a review. Am J Surg. 1994 Nov: 168(5):446-50.

13. McCarthy J. Plastic Surgery. Vol 8 The hand and the upper limb.

14. Wei. F. CH., Mardini. Colgajos en cirugía reparadora.

15. LeedyJE, JanisJE, RohrichRJ. Reconstructionofacquiredscalpdefects: an algorithmic approach. Plast Reconstr Surg 2005:116(4):54e-72e.

16. Zhao J, Song G, Zong X, et al. Using the Reversed Temporal Island Flap to Cover Small Forehead Defects from Titanium Mesh Exposure After Cranial Reconstruction. World Neurosurg. 2018Apr;112:e514-e519.

17. Labow BI, Rosen H, Pap SA, Upton J. Microsurgical reconstruction: a more conservative method of managing large scalp defects? J Reconstr Microsurg 2009;25(8):465-74.

18. Juri J, Juri C. Aesthetic aspects of reconstructive scalp surgery. Clin Plast Surg 1981;8:243.

19. Kwiecien GJ, Rueda S, Couto RA, et al. Long-term outcomes of cranioplasty. Ann Plas Surg 2018;81:416-22.

20. Sosin M, De la Cruz C, Bojovic B, Christy MR, Rodriguez ED. Microsurgical Reconstruction of Complex Scalp Defects: An Appraisal of Flap Selection and the Timing of Complications. J Craniofac Surg 2015 Jun;26(4):1186-91.

21. Baumeister S, Peek A, Friedman A, Scott Levin L, Marcus JR. Management of Postneurosurgical Bone Flap Loss Caused by Infection. Plast Reconstr Surg 2008 Dec;122(6):195e-208e.
22. Pang-Yun Chou. Salvage of postcranioplasty implant exposure using free tissue transfer. Head and Neck journal 2019

23. Nelligan, P. Flap Selection in cranial base reconstruction. Pastic and Reconstructive Surg 1996.

24. Andrea L. Pusic, M. M. Microvascular Reconstruction of the Skull Base: A Clinical Approach to Surgical Defect Classification and Flap Selection. Head and Neck Journal 2007.

25. McCombe D, Donato R, Hofer SO, Morrison W. Free flaps in the treatment of locally advanced malignancy of the scalp and forehead. Ann Plast Surg. 2002:48(6):600-606.

26. St-Hilaire H, Mithani SK, Taylor J, Simmons OP, Singh N, RodriguezED. Restoring the failed cranioplasty: nonanatomical titanium mesh with perforator flap. Plast Reconstr Surg 2009;123(6):1813-7.

27. Dong, et al Latissimus Dorsi-Myocutaneous Flap in the Repair of Titanium Mesh Exposure and Scalp Defect After Cranioplasty. The journal of craniofacial surgery. 2019

28. Kim YC et al. Impact of Venous Outflow Pattern on Flap Compromise in Head and Neck Reconstruction: Review of 309 Radial Forearm Free Flaps. J Craniofacial Surg(2019)

29. Neligan, P. Head and Neck Reconstruction. Plast and Reconstr Surg. Febr 2013

30. Pefaure J. Mackfarlane M. Angrigiani C. Shunt arteriovenoso en cirugía reconstructiva. Revista Iberolatinoamericana 2009;3:456-67.

31. Zhai QK. Proper Choice of Donor Site Veins for Patients Undergoing Free Radial Forearm Flap Reconstruction for the Defects of Head and Neck. J Oral Maxillofac Surg 2018 Mar.

32. Carrell A, Guthrie CC. Complete amputation of the thigh with replan tation. Am J Med Sci 1906;131:297-300.

33. Carrell A. Uniterminal and biterminal venous transplantation. Surg Gynecol Obstet 1906;2:266-9.

34. Carell $A$, Guthrie CC. The reversal of the circulation in a limb. Ann Surg 1906:43:203-15.

35. Calikapan GT, Yildirim S, Akoz T. One-stage reconstruction of large scalp defects: Anterolateral thigh flap. Microsurgery 2006:26:155-9.

36. Hilaire H. Rodriguez E. Restoring the Failed Cranioplasty: Nonanatomical Titanium Mesh with Perforator Flap. Plastic and Reconstructive Surgery 2008;123 (6):1813-7.

37. Tadros M, Costantino PD. Advances in cranioplasty: A simplified algo rithm to guide reconstruction of acquired defects. Facial Plast Surg. 2008:24:135-45.

38. Serra MP, Longhi P, Carminati M, Righi B, Robotti E. Microsurgical scalp and skull reconstruction using a combined flap composed of serratus anterior myo-osseous flap and latissimus dorsi myocutaneous flap. Journal of Plastic, Reconstructive \& Aesthetic Surgery 2007;60:1158e.e1161.

39. Chang KP, Lai CH, Chang CH, et al. Free flap options for reconstruction of complicated scalp and calvarial defects: report of a series of cases and literature review. Microsurgery 2010;20:13-8.

40. Calikapan GT, Yildirim S, Akoz T. One-stage reconstruction of large scalp defects: anterolateral thigh flap. Microsurgery 2006;26:155-9. 